\title{
From the Roemer mission to Gaia
}

\author{
E. $\mathbf{H} \varnothing \mathrm{g}$ \\ Niels Bohr Institute, Copenhagen University, Juliane Maries Vej 30, 2100 Copenhagen Ø, \\ Denmark \\ email: erik@astro.ku.dk
}

\begin{abstract}
At the IAU symposium in Shanghai September 1992 the present author made the first proposal for a specific mission concept post-Hipparcos, the first scanning astrometry mission with CCDs in time-delayed integration mode (TDI). Direct imaging on CCDs in long-focus telescopes was described as later adopted for the Gaia mission. The mission called Roemer was designed to provide accurate astrometry and multi-colour photometry of 400 million stars brighter than $18 \mathrm{mag}$ in a five-year mission. The early years of this mission concept are reviewed.
\end{abstract}

Keywords. space vehicles, astrometry, instrumentation: photometers

When Hipparcos was launched in August 1989 the Hipparcos Science Team (HST) was present in Kourou and we were greatly relieved seeing the take-off after the many years of preparation. But that changed to grim disappointment the next day when we learned that the apogee boost motor had not started so that the satellite was stuck in an elliptical transfer orbit instead of the intended geostationary. This endangered the whole mission and we would possibly only get a much shorter set of poor observations, perhaps only months and not the planned three years. Passing through the radiation belts every few hours could soon destroy the electronics and solar cells.

In this mood, but optimistic as always, I presented the Hipparcos mission on behalf of Michael Perryman who could not be present, and the Tycho project at the IAU Symposium No.141 October 17-21, 1989 in Leningrad (now St. Petersburg). The audience was full of high hopes for Hipparcos - hopes which were in fact justified as we should later see. We noticed that Soviet (later Russian) colleagues presented ideas at the Symposium for a successor to Hipparcos. They themselves had three projects on the drawing boards: AIST/STRUVE, LOMONOSOV, and REGATTA-ASTRO. The basic idea was to reobserve the 120000 Hipparcos stars and utilize the positions from Hipparcos and those from a new epoch to get much better proper motions than Hipparcos alone would achieve, even if its severe problems would be cured.

Such ideas were far beyond the horizon of anyone in the Hipparcos team, busy as we were to get our mission to work and to perform the very complex data analysis. I was myself leader of one of the two Hipparcos data analysis teams and of the Tycho team and thus had more than enough to look after.

Shortly later I was invited to lecture about Hipparcos at the Pulkovo Observatory in Leningrad, the Mission Control Center in Moscow, and the Kislovodsk Observatory in Caucasus. I was accompanied on the journey in August 1990 by M.S. Chubey, V.V. Makarov, and V.N. Yershov so we had plenty of time for discussions. I wanted to understand how their AIST project functioned, but unexpectedly, after a day I was more occupied by designing a second Hipparcos myself, realizing that it could easily be made ten times more efficient in utilizing the star light, mainly by employing more detectors, while keeping the same telescope aperture of $0.29 \mathrm{~m}$.

In June 1991 an International Symposium "Etalon" Satellites was held in Moscow where I presented a paper with Mark Chubey "Proposal for a second Hipparcos", but the 
proceedings were not published. If launched ten years after Hipparcos the mission could obtain proper motions for the 120000 Hipparcos stars with an accuracy 10 times better than expected from Hipparcos as well as 1 mas accuracy for all astrometric parameters of some 400000 stars and four-colour photometry for two million stars. This proposal was considered by the Mission Control Centre in Moscow.

During 1990-91 we met many times for discussion of our ideas as they developed, and Lennart Lindegren joined us. At the HST meetings I got a few minutes to present the progress, somewhat reluctantly allowed by the chairman who probably thought we had enough to do with one mission for the time being.

In 1991 I had left the study of photon counting techniques as in Hipparcos and tried to use CCDs, a completely new technique for me. I learnt it from our engineer, R. Florentin Nielsen, and designed a detector system using a modulating grid as in Hipparcos. The result was 1000 times better light efficiency than Hipparcos (see Høg \& Lindegren in IAU Symposium 156, 1993).

Having done that I dropped the modulating grid and tried direct imaging on the CCDs imployed in drift-scan mode or time-delayed integration (TDI). That design was called Roemer and gave 100000 times better light efficiency with the same telescope aperture $(0.29 \mathrm{~m})$, but a very long focal length was needed, $5 \mathrm{~m}$ instead of the $1.4 \mathrm{~m}$ in Hipparcos (see Høg 1993). Both systems were presented at the IAU Symposium 156 in Shanghai September 15-19, 1992.

The Roemer design was proposed in June 1993 for the Third Medium Size ESA Mission (M3) by a team mainly from the HST. The proposal got a high rating in the ESA selection committee, but was not finally selected because it was considered to come too early after Hipparcos. This view was not shared by the proposers, but in hindsight it was a wise decision because it gave us time for much development in the subsequent years.

Interferometry was proposed at the IAU Symposium No. 166 in August 1994 by Lindegren \& Perryman "A small interferometer in space for global astrometry: The Gaia concept", stating the "very strong scientific case for global optical astrometry at the 20 microarcsec accuracy level." The satellite should contain three Fizeau-type interferometers with $2.5 \mathrm{~m}$ baselines.

At the same IAU Symposium a 10 microarcsec mission (Roemer+) with 9-colour intermediate- and wide-band filter photometry was proposed by the present author. The better performance was obtained with two telescopes of larger apertures of $70 \mathrm{~cm}$ instead of $29 \mathrm{~cm}$. Picometer gauges were adopted to monitor the alignment of the telescopes.

The development of instrument ideas had mainly three scientific goals: higher astrometric accuracy of 10 microarcsec instead of the 100 microarcsec envisaged in Roemer, measurement of radial velocities for the brighter stars with the satellite, and better multicolour photometry. These improvements were considered crucial for an ambitious ESA mission aiming for understanding the details of our Galaxy. Thorough assessment of the scientific goals and the data analysis was also made. - Finally, Gaia is now scheduled for launch in 2011 on a 5 year mission to measure 1000 million stars brighter than 20 mag.

\section{Why interferometry - and why not?}

There was a widespread belief at the time of the Roemer proposal, Lindegren et al. (1993), that interferometry could give better astrometry from space, and a section was included: "Towards 10 microarcsec astrometry: The FIZEAU option". It was not part of the baseline Roemer proposal, but was meant "to point out a possible development towards a scanning satellite with ten times the angular accuracy of Roemer", and the enourmous scientific benefits of such an accuracy for millions of stars were outlined. 
A Fizeau principle was subsequently used in several proposals for scanning astrometric satellites, e.g., the Gaia concept of 1994 mentioned above, FAME and DIVA.

I agreed that interferometric options should be deeply studied as they in fact were during the following years. Perhaps the complications of interferometry could be alleviated, or at least the fallout from studies could bear fruit in other (unforeseen) contexts. These studies always focussed on a scanning astrometry satellite similar to Hipparcos because a systematic scanning of the sky was considered the only way to measure the millions of stars required for our scientific goal. A pointing satellite could never do that, but would of course have the advantage of allowing longer integration time on any selected area.

My own preference in instrument design has always been to identify and focus on difficulties and try to solve or circumvent them. So I believed more in direct imaging on CCDs from full-aperture telescopes than in the diluted apertures required for interferometry. The Roemer+ design of 1994 used full apertures and obtained 10 microarsec, but it required picometer gauges to monitor the alignment of telescopes, a technique nearly always required in interferometric options.

In 1995 we designed an interferometric option later published by Høg et al. (1997). It used a beam-combiner of $150 \mathrm{~cm}$ aperture and a simple telescope, basically an aplanatic Gregorian system. A prism provided a low dispersion perpendicular to the scanning direction so that spectrophotometry could be obtained. This new option of Gaia was adopted in ten times smaller size for the proposal by Röser et al. (1997). This was a small German astronomy satellite, DIVA, planned for launch in 2003 to measure about 40 million stars as a fore-runner for Gaia. But funding did not follow suit.

The ESA studies of the interferometric option are described at length in a section (pp.331-338) of ESA (2000) and complete references are given. The history of the development of Gaia is briefly summarized in Perryman et al. (2001). One of the problems was that the split pupil of an interferometer did not allow accurate measurement of the stars about 20 mag required for the ambitious scientific goal, but only about 17 mag. Another problem came from the required data rate to be transmitted from the satellite. An interferometric image requires a lot more data points to cover the fringes of a star than a direct stellar image from a full aperture. The higher data rate could well be accepted from a geostationary orbit, but the thermal control during eclipses would jeopardize the instrument stability, so the orbit around L2 was required for thermal stability. Here the data rate of one Megabit per second for the full aperture option was acceptable, but not the higher rate for interferometry. Other problems of interferometry were indentified and in the end the full aperture could be selected and we were sure that all had been done to investigate both options, based on industrial studies by Matra Marconi Space for the baseline design and Alenia Aerospazio for the interferometric.

\section{References}

ESA 2000 Gaia: Composition, Formation and Evolution of the Galaxy Technical Report ESA$\mathrm{SCI}(2000) 4$

$\mathrm{H} \varnothing \mathrm{g}$ E. 2007, This poster on 6 pages, including the complete list of references at www.astro.ku.dk/ erik/ShanghaiPoster.pdf, and some slides at www.astro.ku.dk/ erik/ ShanghaiHoeg.pdf

IAU Symposium No. 141, 1990, J. H. Lieske \& V. K. Abalakin (eds.) Inertial Coordinate System of the Sky.

IAU Symposium No. 156, 1993, I. I. Mueller \& B. Kolaczek (eds.) Developments in Astrometry and their Impact on Astrophysics and Geodynamics.

IAU Symposium No. 166, 1995, E. Høg \& P. K. Seidelmann (eds.) Astronomical and Astrophysical Objectives of sub-milliarcsecond Optical Astrometry. 